Nota de investigación

\title{
Efectividad de fungicidas y Trichorderma spp. para el control de Lasiodiplodia spp. en huertos de limón 'Persa' en Veracruz
}

\author{
Santos Gerardo Leyva-Mir ${ }^{1}$ \\ Marco Antonio Bautista-Cruz ${ }^{2}$ \\ Gustavo Almaguer-Vargas ${ }^{2}$ \\ María Teresa Colinas-León ${ }^{2}$ \\ Juan Manuel Tovar-Pedraza ${ }^{3}$ \\ Moisés Camacho-Tapia ${ }^{4 \S}$
}

${ }^{1}$ Departamento de Parasitología Agrícola-Universidad Autónoma Chapingo. Carretera México-Texcoco km 38.5, Texcoco, Estado de México, México. CP. 56230. Tel. 5959521500, ext. 6179. (1santos@correo.chapingo.mx). ${ }^{2}$ Posgrado en Horticultura-Departamento de Fitotecnia-Universidad Autónoma Chapingo. Carretera México-Texcoco km 38.5. Texcoco, Estado de México, México. CP. 56230.

Tel. 595 9521500, ext. 6390. (bacma_74@hotmail.com; almaguervargas@ @otmail.com; lozcol@gmail.com). ${ }^{3}$ Laboratorio de Fitopatología, Coordinación Culiacán, Centro de Investigación en Alimentación y Desarrollo. Carretera El Dorado Campo El diez km 5.5, Sinaloa, México. CP. 80110. Tel. 6674806950 (juan.tovar@ciad.mx). ${ }^{4}$ Laboratorio Nacional de Investigación y Servicio Agroalimentario y Forestal-Universidad Autónoma Chapingo. Carretera México-Texcoco km 38.5, Texcoco, Estado de México, México. CP. 56230. Tel. 595 9521500, ext. 6450.

${ }^{\S}$ Autor para correspondencia: moises.camachotapia@gmail.com.

\section{Resumen}

La muerte descendente del limón 'Persa' (Citrus latifolia Tan.) causada por Lasiodiplodia spp., es una enfermedad de alta importancia. La intensidad de las podas en limón incrementa la susceptibilidad y la pérdida anual de hasta 35\% de árboles, lo que resulta $60 \%$ de reducción de la producción. Durante los años 2016 y 2017, en el municipio de Papantla, Veracruz se evaluó la efectividad del control químico y biológico contra Lasiodiplodia spp., después de la poda. Se realizó un diseño de bloques completo al azar, se aplicaron cinco tratamientos: metil tiofanato ( $1 \mathrm{~g}$ $\left.\mathrm{L}^{-1}\right)$, tiabendazole $\left(2.5 \mathrm{~g} \mathrm{~L}^{-1}\right)$, clorotalonil $\left(3 \mathrm{~g} \mathrm{~L}^{-1}\right)$ y mancozeb $\left(4 \mathrm{~g} \mathrm{~L}^{-1}\right)$, Trichoderma harzianum + Trichoderma viridae $\left(20 \mathrm{ml} \mathrm{L}^{-1}\right)$ y un testigo (sin aplicación). Se realizaron dos aspersiones dirigidas a tronco, ramas y follaje hasta punto de goteo. Se evalúo la incidencia y severidad de la enfermedad cada siete días después de la primera aplicación. Con los datos de incidencia se realizó ajuste de modelos epidemiológicos y se calcularon los parámetros descriptivos como: intensidad inicial, tasa de incremento y área bajo la curva del progreso de la enfermedad. El fungicida metil tiofanato tuvo mayor efectividad para el control de la enfermedad, seguido de tiabendazol.

Palabras claves: Lasiodiplodia spp., control de la enfermedad, muerte descendente.

Recibido: enero de 2021

Aceptado: febrero de 2021 
El limón 'Persa' (Citrus latifolia Tan.) pertenece a la familia Rutaceae y en México el área con mayor producción de este frutal está situada en el Golfo (Mendoza-Tornez et al., 2016). Entre los factores que reducen la producción de limón 'Persa' se encuentran diversas enfermedades, incluyendo al virus de la tristeza, exocortis, huanglongbing, antracnosis, muerte descendente, gomosis entre otras. Los daños por gomosis en cítricos son ocasionados por Phythopthora spp. Para lo cual se recomienda la aplicación de fungicidas como fosetil-Al y metalaxil (Jadeja et al., 2000; Raina, 2012; Acosta-Pérez et al., 2014).

Sin embargo, en algunos casos, estas aplicaciones no tienen resultados satisfactorios al controlar la gomosis, ya que Lasiodiplodia spp., también inducen gomosis en las áreas afectadas y generalmente son confundidas con Phytophthora spp. Dicha situación se presenta en las zonas productoras de Veracruz, donde se disminuye la vida productiva y producción en árboles de limón 'Persa'. En 2019 se reportó a L. pseudotheobromae, L. theobromae, L. brasiliense, L. subglobosa, L. citricola y L. iraniensis como los agentes causales de la muerte descendente y cancros en huertos comerciales de limón 'Persa' en Puebla y Veracruz (Bautista-Cruz et al., 2019).

En 2019 en Morelos fue reportado de igual forma a L. citricola, L. theobromae y $L$. pseudotheobromae afectando a limón 'Persa' (Valle-de la Paz et al., 2019a). Asimismo, PolancoFlorian et al. (2019) reportaron como causante de la muerte descente en naranjo dulce de Nuevo León y Tamaulipas a L. theobromae, Fomitopsis meliae y Eutrypella citricola.

Los hongos del género Lasiodiplodia son fitopatógenos inespecíficos, pueden sobrevivir como endófitos y como saprófitos bajo condiciones de estrés abiótico. Se desarrollan en regiones subtropicales y tropicales donde afectan a más de 1100 hospedantes leñosos, causando enfermedades como la muerte descendente, gomosis y pudrición del pedúnculo (McDonald et al., 2011; Al-Sadi et al., 2013; Coutinho et al., 2017). Asimismo, Lasiodiplodia spp., se han reportado induciendo síntomas de muerte descedente, gomosis y cancros en amplio rango de especies de cítricos (Al-Sadi et al., 2013; Coutinho et al., 2017).

Lasiodiplodia spp. causan la muerte descendente y gomosis, caracterizada por necrosis de corteza y madera, así como cancros en tallos en huertos de limón 'Persa' reportados en el estado de Veracruz, en la región de Martínez de la Torre, Tlapacoyan y Papantla y en el estado de Puebla en Acateno y Hueytamalco (Bautista-Cruz et al., 2019). Estos hongos fitopatógenos disminuyen la productividad de los cítricos, $60 \%$ y la pérdida anual de árboles puede llegar hasta un 35\%. Algunos estudios reportaron la efectividad de fungicidas como metil tiofanato para el control de la muerte descendente de huertos de mango provocada por Lasiodiplodia spp. (Khanzada et al., 2005; Shahbaz et al., 2009; Naqvi et al., 2015).

Otros estudios, reportaron la efectividad de Trichoderma para el control de enfermedades fungosas en cítricos (García et al., 2011; El- Mohamedy et al., 2012). En el caso del mango, se ha reportado que el principal punto de acceso de Lasiodiplodia spp., es a través de las heridas de poda, daños causados por insectos, ramas quebradas por el viento o por exceso de carga de fruta (Sakadilis et al., 2011). En el cultivo de limón 'Persa' se realizan podas intensas como parte del manejo del cultivo, y esto favorece la incidencia de gomosis, por lo que es necesario proteger a los árboles inmediatamente después de realizar la poda. Por esta razón, el objetivo de esta investigación fue evaluar la efectividad de productos químicos y biológicos, después de realizar la poda, para el manejo de la muerte descendente en huertos comerciales de limón 'Persa'. 
Se seleccionó un huerto de limón 'Persa' injertados en Citrus volkameriana, en el ejido de Insurgente Socialista, del municipio de Papantla, estado de Veracruz, México y donde previamente se identificaron seis especies de Lasiodiplodia (Bautista-Cruz et al., 2019), los árboles de limón tenían ocho años de edad y presentaban una distancia de plantación de 5 x $5 \mathrm{~m}$. En el huerto seleccionado, durante los años 2016 y 2017, se evaluó la efectividad de los siguientes productos: metil tiofanato, tiabendazole, clorotalonil, mancozeb (Cuadro 1), un producto a base de Trichoderma harzianum +Trichoderma viridae a una concentración de $8 \times 10^{8}$ conidios por ml y por último un control, donde sólo se aplicó agua, lo que dio un total de seis tratamientos establecidos en campo bajo un diseño de bloques completos al azar.

Cuadro 1. Productos químicos para el control de Lasiodiplodia spp. en limón 'Persa'.

\begin{tabular}{cccc}
\hline Ingrediente activo & Modo de acción & IA $(\%)$ & Dosis $\left(\mathrm{g} \mathrm{L}^{-1}\right)$ \\
\hline Metil tiofanato & Sistémico & 70 & 1 \\
Tiabendazol & Sistémico & 60 & 2.5 \\
Maconzeb & Contacto & 80 & 3 \\
Clorotalonil & Contacto & 75 & 4 \\
\hline
\end{tabular}

Cada tratamiento tuvo seis árboles y de cada árbol se podaron dos ramas con un diámetro promedio de $3.5 \mathrm{~cm}$, dejando una longitud final de $20 \mathrm{~cm}$ y que estuvieran ubicadas en cada punto cardinal, dando un total de 48 unidades de muestreo. Después de podar las ramas, se aplicaron los tratamientos con un aspersor motorizado SR 420 (Sthil, México) a cada árbol de limón hasta punto de goteo para asegurar la cobertura del producto. A los 20 días posteriores a la primera aplicación se realizó una segunda aplicación. Se realizaron siete evaluaciones semanales, las cuales iniciaron después de haber transcurrido siete días de la primera aplicación de los tratamientos.

La variable respuesta fue la longitud de las lesiones de Lasiodiplodia en las ramas podadas. Las medidas obtenidas se utilizaron para poder caracterizar la severidad de la enfermedad. Con los datos de las evaluaciones se realizaron ajustes de modelos epidemiológicos que describieron el desarrollo de la enfermedad a través del tiempo. Con ayuda del paquete estadístico SAS versión 9.2 (SAS, 2008), se analizaron los modelos epidemiológicos (Exponencial, Monomolecular, Logístico y Gompertz).

La selección del modelo descriptor de la enfermedad fue con el coeficiente de determinación $\left(\mathrm{R}^{2}\right)$. Además, se determinaron parámetros de la epidemia como intensidad inicial de la enfermedad ( $\left.\mathrm{Y}_{0}\right)$, tasa de incremento de la enfermedad (\%·día) y área bajo la curva del progreso de la enfermedad (ABCPE) (\%-día) mediante el método de integración trapezoidal (Campbell y Madden, 1990).

Para determinar el efecto de los tratamientos y comparar cada uno de los parámetros epidemiológicos se realizó un análisis de varianza $(\alpha=0.05)$. Asimismo, se realizó una comparación de medias mediante la prueba de diferencia mínima significativa (Fisher, $\alpha=0.05$ ). Para todos los análisis se utilizó el paquete estadístico SAS versión 9.2. Los tratamientos empleados disminuyeron el avance de los síntomas de la muerte descendente, mientras que los árboles testigo, presentaron un desarrollo evidente de la enfermedad. El modelo que mejor describió la epidemia fue el logístico $\left(\mathrm{y}=1 /\left(1+\left(\left(1-\mathrm{y}_{0}\right) / \mathrm{y}_{0}\right) * \operatorname{ex}(-\mathrm{rL} * \mathrm{t})\right)(\right.$ Cuadro 2$)$. 
Cuadro 2. Efecto de los tratamientos en los parámetros descriptivos del desarrollo de Lasiodiplodia spp. en limón 'Persa'.

\begin{tabular}{cccc}
\hline Tratamiento & $\mathrm{Y}_{0}$ & $\mathrm{rL}$ & $\mathrm{ABCPE}$ \\
\hline Metil tiofanato & $0.03008 \mathrm{~B}$ & $0.04536 \mathrm{~F}$ & $514.90461 \mathrm{~F}$ \\
Tiabendazol & $0.030541 \mathrm{~A}$ & $0.049764 \mathrm{E}$ & $4380.19606 \mathrm{E}$ \\
Trichoderma spp. & $0.022177 \mathrm{C}$ & $0.078434 \mathrm{D}$ & $15374.4392 \mathrm{D}$ \\
Maconzeb & $0.00871 \mathrm{D}$ & $0.114338 \mathrm{C}$ & $59421.3886 \mathrm{~B}$ \\
Clorotalonil & $0.00137 \mathrm{E}$ & $0.164006 \mathrm{~B}$ & $37380.0589 \mathrm{C}$ \\
Testigo & $0.000757 \mathrm{~F}$ & $0.223954 \mathrm{~A}$ & $336936.28 \mathrm{~A}$ \\
\hline
\end{tabular}

$\mathrm{Y}_{0}=$ intensidad inicial de la enfermedad; $\mathrm{rL}=$ tasa de incremento de la enfermedad (\%-día); $\mathrm{ABCPE}=$ área bajo la curva del progreso de la enfermedad (\%-día).

Se observó que en todos los tratamientos la intensidad inicial de la enfermedad ( $\mathrm{Y}_{0}$ ) presentó algunas variaciones, indicando una intensidad inicial menor en el testigo. Esta situación se pudo deber a las condiciones naturales en las que se estableció el experimento, ya que en campo la variación del inóculo podría deberse a la distribución espacial de los árboles evaluados, además en este estudio no se realizó inoculación directa de Lasiodiplodia spp., sino que se esperó a que la infección ocurriera por condiciones naturales considerando el principio de contagio a través de inóculo en el suelo y ramas circundantes que tuvieran síntomas y esporas que incrementaran la intensidad inicial de la enfermedad. Sin embargo, con las aplicaciones de los tratamientos la intensidad inicial de inóculo pudo ser reducida para conducir un menor desarrollo de la epidemia.

Al realizar una segunda aplicación a los 20 días, se puede garantizar la reducción del inóculo generado después del inicio del experimento, y esto tiene un efecto directo en la tasa de incremento (rL). Considerando esto, se puede indicar que el mejor producto para controlar Lasiodioplodia spp., es el metil tiofanato, ya que redujo la rL (0.04536\%.día) y se observó al presentar una ABCPE (514.90461 \%-día) menor en comparación a los demás tratamientos; que, como parámetro integrativo en la descripción del comportamiento de la enfermedad, proporcionó un entendimiento de la importancia de reducir la tasa de incremento y su efecto en el desarrollo de una enfermedad.

El tiabendazol tuvo control después del metil tiofanato, por lo cual podrían considerarse como una alternativa para el control de la enfermedad. En este mismo sentido, se podría considerar el tiabendazol para una segunda aplicación, ya que tiene efecto en reducir la tasa de incremento, en este estudio presentó una rL de 0.049764 , la cual es similar a la presentada por el metil tiofanato. El testigo exhibió una tasa de incremento de $0.223954 \%$, que resultó en una $\mathrm{ABCPE}$ de 336936.28\%-día, que describió un desarrollo evidente de la epidemia.

Los fungicidas de contacto clorotalonil y mancozeb tuvieron una mayor tasa de incremento, 0.164006 y $0.114338 \%$. día respectivamente, que dio como resultado una mayor ABCPE, por lo tanto, estos fungicidas no deberían considerarse para el manejo curativo de Lasiodiplodia spp. Valle-de la Paz et al. (2019b) reportan que aislados L. theobromae y L. citricola en limón 'Persa' de Morelos, México y en condiciones in vitro, son sensibles a metil tiofanato y tiabendazol, lo que concuerda con esta investigación en campo, mientras que en condiciones in vitro ellos reportan un buen control con el uso de Trichoderma pero en condiciones de campo nosotros no vimos un buen control. 
De manera similar, Masood et al. (2014), reportaron alta efectividad para el control de la muerte descendente en cultivo de mango en Pakistán, con el uso de metil tiofanato, mediante la inyección al xilema y aplicaciones foliares. Por otra parte, Khanzada et al. (2005) encontraron que el carbendazim tuvo mayor efecto que el metil tiofanato, al ser evaluado en el mismo patosistema. En el caso de mango, al inyectar el metil tiofanato directo al xilema tres meses después de iniciado el tratamiento, reduce la defoliación en los árboles. Sin embargo, no se consigue eliminar las lesiones con presencia de gomosis.

Entretanto, Khanzada et al. (2005) al aplicar carbendazim, tiofanato de metilo y fosetil aluminio para el control de la muerte descendente del mango en Pakistán, encontraron efectividad aceptable de tiofanato de metilo en el control de esta enfermedad (solo superado por carbendazim) y además de eso, con este estudio, logró reducir la infección por hongos, suprimió la exudación de la goma, la muerte y el marchitamiento de ramas de mango, además de que se notó un aumento significativo en el crecimiento vegetativo de las plantas. En naranja Valencia tardía se reportó alta efectividad de tiabendazol para el control de Guinardia citricarpa (Yan et al., 2016).

Por otra parte, Tovar-Pedraza et al. (2013) lograron disminuir hasta $62 \%$ la incidencia de la muerte descendente en la fase de injertación en viveros de zapote mamey, al tratar varetas por inmersión con tiabendazol y mancozeb, ellos reportaron que tiabendazol mostró mayor efectividad en el control de la enfermedad.

De igual forma Tovar-Pedraza et al. (2013) mencionan que la combinación de fungicidas y lavado previnieron la infección por $L$. theobromae durante el proceso de injerto en zapote mamey. Shahbaz et al. (2009); Navqui et al. (2015) reportaron que en condiciones in vitro el metil tiofanato tiene alta efectividad en el control de Lasiodiplodia. Wang et al. (2007) indicaron que procloraz, iprodiona y tebuconazol fueron los fungicidas más efectivos para inhibir el crecimiento micelial de L. theobromae en papaya, de igual forma Bester (2007) reportó el uso de procloraz y tebuconazol para el control de Lasiodiplodia en uva.

En este trabajo Trichoderma no tuvo un buen control, esto pudo deberse a que se aplicó de forma foliar, en comparación con otros investigadores donde lo aplican en suelo para controlar patógenos que afectan a raíz, además no se garantizaron las condiciones óptimas para el desarrollo de este agente de control (Harman et al., 2004; Infante et al., 2009; Martínez et al., 2013).

Algunos estudios en limón 'Persa' y tangerina indicaron que Trichoderma spp., puede tener un efecto similar que el metil tiofanato para controlar la pudrición de la raíz ocasionada por Fusarium (El-Mohamedy et al., 2012; El-Mohamedy et al., 2013). En estudios realizados in vitro, Bhadra et al. (2015) reportaron alta efectividad de T. viridae en el control de Lasiodiplodia theobromae.

Considerando los resultados obtenidos en este experimento, no se recomendaría el uso de Trichoderma para el control de Lasiodiplodia, debido a que la infección está dada en las ramas y el agente de control biológico al ser aplicado en este lugar, no tiene todas las condiciones necesarias para desarrollarse y estar presente para el control Lasiodiplodia.

En esta investigación se observó que el clorotalonil tuvo mejor efecto hasta los 28 días, ya que redujo el desarrollo de la enfermedad de forma más eficiente. Después de 28 días, este producto pierde su efectividad, mientras que el metil tiofanato tiene un control durante todo el tiempo de 
evaluación. Mancozeb fue el fungicida menos efectivo en este experimento. En los árboles tratados con metil tiofanato se observó mayor cicatrización de esas lesiones seguido de tiabendazol, Trichoderma spp., clorotalonil y mancozeb, en comparación con los árboles no tratados. Sin embargo, después de 45 días de la primera aplicación (ddpa), algunas de las lesiones que ya estaban cicatrizando, comenzaron a presentar poco escurrimiento de goma. En los árboles no tratados, la enfermedad se incrementó con el transcurso del tiempo, hasta que algunos de los tocones colapsaron.

En la región de estudio se observa que algunas prácticas como conservar el limón en el árbol hasta que incremente su valor, son causa del aumento de la incidencia de Lasiodiplodia y pudrición del pedúnculo. Además los frutos momificados y los restos de poda que se dejan en el suelo son fuente de inóculo, que impacta en la intensidad inicial de la epidemia, además de la tasa de incremento en los ciclos de reinfección en los árboles, para evitar esto, es recomendable realizar las aplicaciones de fungicidas necesarias, además de retirar residuos de poda y evitar dejar los fruto en los árboles por tiempo innecesario, ya que repercute además en el incremento en la susceptibilidad de los árboles a la infección por Lasiodiplodia.

Generalmente se asocia a Phythopthora spp., como el agente causal de la gomosis de los cítricos, y para su control se realizan tratamientos a base de metalaxil y fosetil-Al (Farih et al., 1981; Jadeja et al., 2000; Raina et al., 2012). En la zona de estudio de Martínez de la Torre, Tlapacoyan, Papantla, se aplica regularmente fosetil-Al y metalaxil para el control de la gomosis, pero no se tienen resultados favorables, ya que la incidencia de gomosis en los huertos superó $92.5 \%$ y las pérdidas económicas se incrementan considerablemente. En la presente investigación, se encontró que el metil tiofanato es el mejor producto para controlar Lasiodiplodiodia, seguido de tiabendazol.

\section{Conclusiones}

El fungicida que tuvo mejor efectividad en el control de la muerte descendente del limón 'Persa' en condiciones de campo, fue metil tiofanato, seguido de tiabendazol. Al realizar dos aplicaciones de fungicidas reduce significativamente la muerte descendente; sin embargo, se sugiere que en un manejo integrado del limón 'Persa', considerar las condiciones climáticas en que se realiza la poda y la aplicaciones para disminuir la fuente de inoculo y la tasa de incremento, ya que en temporada de lluvias se incrementaría incidencia y severidad de Lasiodiplodia spp.

\section{Literatura citada}

Acosta-Pérez, J. A.; Ortíz-Gacía, C. F.; Zaldívar-Cruz, J. M.; Rodríguez-Cuevas, M.; BautistaMuñoz, C. C. y Castillo-Aguilar, C. 2012. Identificación del agente causal e importancia de la gomosis en la región citrícola de Huimanguillo, Tabasco, México. México. Universidad y Ciencia Trópico Húmedo. 28(3):245-258. http://www.scielo.org.mx/ scielo.php?script=sci_arttext\&pid=S0186 29792012000300004 .

Al-Sadi, A. M.; Al-Wehaibi, A. N.; Al-Shariqi, R. M.; Al-Hammadi, M. S.; Al-Hosni, I. A.; AlMahmooli, I. H.; and Al-Ghaithi, A. G. 2013. Population genetic analysis reveals diversity in Lasiodiplodia species infecting date palm, Citrus, and mango in Oman and the UAE. USA. Plant Disease. 97(10):1363-1369. https://doi.org/10.1094/PDIS-03-13-0245-RE. 
Bautista-Cruz, M. A.; Almarguer-Vargas, G.; Leyva-Mir, S. G.; Colinas-León, M. T.; Correia, K. C.; Camacho-Tapia, M.; Robles-Yerena, L.; Michereff, S. J. and Tovar-Pedraza, J. M. 2019. Phylogeny, distribution, and pathogenicity of Lasiodiplodia species associated with cankers and dieback symptoms of persian lime in Mexico. USA. Plant Dis. 103(6):11561165. https://doi.org/10.1094/PDIS-06-18-1036-RE.

Bester, W.; Crous, P. W. and Fourie, P. H. 2007. Evaluation of fungicides as potential grapevine pruning wound protectants against Botryosphaeria species. Australia. Australasian Plant Pathology. 36(1):73-77. https://doi.org/10.1071/AP06086.

Bhadra, M. M; Khair, A.; Hossain, M. A. and Sikder, M. M. 2015. Efficacy of Trichoderma spp. and fungicides against Lasiodiplodia theobromae. Bangladesh. Bangladesh J. Sci. Industrial Res. 49(2):125-130. https://doi.org/10.3329/bjsir.v49i2.22008.

Campbell, C. L. and Madden, L. V. 1990. Introduction to plant disease epidemiology. John Wiley and Sons Inc. New York. 532 p.

Coutinho, I. B. L.; Freire, F. C. O.; Lima, C. S.; Lima, J. S.; Gonçalves, F. J. T.; Machado, A. R.; Silva, A. M. S. and Cardoso, J. E. 2017. Diversity of genus Lasiodiplodia associated with perennial tropical fruit plants in northeastern Brazil. USA. Plant Pathol. 66(1):90-104. https://doi.org/10.1111/ppa.12565.

Donald, V. and Eskalen, A. 2011. Botryosphaeriaceae species associated with avocado branch cankers in California. USA. Plant Dis. 95(11):1465-1473. https://doi.org/10.1094/PDIS02-11-0136

El-Mohamedy, R. S. R.; Morsey, A. A. and Bakeer, A. R. T. 2013. Utilization of bio composted agricultural wastes in management of Fusarium dry root rot disease on lime (Citrus aurantifolia L.). Thailand. J. Agric. Technol. 9(5):1215-1225. http://www.ijataatsea.com/pdf/v9_n5-13-September/17-Plant\%20Protection\%20-IJAT-2013-9(5)-S.\%20 Safari-M.Javad\%20Soleimani\%20-KS.pdf.

El-Mohamedy, R. S. R.; Morsey, A. A.; Diab, M. M.; Abd-Kareem, F. and Faraag, E. S. 2012. Management of dry root disease of mandarin (Citrus reticulate Blanco) through biocomposted agricultural wastes. Thailand. J. Agric. Technol. 8(3):969-981. https://www.semanticscholar.org/paper/Management-of-dry-root-rot-disease-ofmandarin-El-MohamedyMorsey/e779a3bb957ccd22c6c9befe53b0e2e440c91fa6.

Farih, A.; Menge, J. A.; Tsao, P. H. and Ohr, H. D. 1981. Metalaxyl and efosite aluminium for control of Phytophthora gummosis and root rot on citrus. USA. Plant Dis. 65(8):654-657. https://doi.org/10.1094/PD-65-654.

García, A.; Rodríguez, K.; Puente, A.; Valero, L. and Rodríguez, G. 2011. Evaluación de alternativas para disminuir fitopatógenos del suelo en áreas de replantación de cítricos. Cuba. Centro Agrícola. 38(4):5-7. http://cagricola.uclv.edu.cu/descargas/pdf/V38Numero_4/cag024111813.pdf.

Harman, G. E.; Howell, C. R.; Viterbo, A.; Chet, I. and Lorito, M. 2004. Trichoderma species opportunistic, avirulent plant symbionts. London. Nature Reviews Microbiology. 2(1):4356. https://www.nature.com/articles/nrmicro797.

Infante, D.; Martínez, B.; González, N. y Reyes, Y. 2009. Mecanismos de acción de Trichoderma frente a hongos fitopatógenos. Cuba. Revista de Protección Vegetal. 24(1):14-21. http://scielo.sld.cu/scielo.php?script=sci-arttext\&pid=S1010-27522009000100002.

Jadeja, K. B.; Mayani, N. G.; Patel, V. A. and Ghodasara, M. T. 2000. Chemical control of canker and gummosis of citrus in Gujarat. Indian. J. Mycol. Plant Pathol. 30(1):87-88. https://www.cabdirect.org/cabdirect/abstract/20001006754. 
Khanzada, M. A.; Lodhi, A. M. and Shahzad, S. 2005. Chemical control of Lasiodiplodia theobromae, the causal agent of mango decline in Sindh. Pakistan. Pakistan J. Bot. 37(4):1023. http://www.pakbs.org/pjbot/PDFs/37(4)/PJB37(4)1023.pdf.

Martínez, B.; Infante, D. y Reyes, Y. 2013. Trichoderma spp. y su función en el control de plagas en los cultivos. Cuba. Revista Protección Vegetal. 28(1):1-11. http://scielo.sld.cu/pdf/rpv/v28n1/rpv01113.pdf.

Masood, A.; Salman, M. and Saeed, S. 2014. Fungicide injection, an efficient management technique of mango sudden death disease in Punjab, Pakistan. Pakistan. Pakistan J. Phytopathol. 26(2):259-263. https://www.pjp.pakps.com/index.php/PJP/article/view/74.

Mendoza-Tornez, R.; Marquez-Berber, S. R.; Almaguer-Vargas, G.; Aguilar-Avila, J.; Gardezi, A. K. and Ayala-Garay, A. V. 2016. Value network of the Persian lime in Mexico. Bosnia and Herzegovina. AGROFOR Inter. J. 1(3):39-45. http://dx.doi.org/10.7251/ AGRENG1603039T.

Naqvi, S. A. H.; Latif, M. R.; Khan, S. A.; Malik, M. T. and Freed, S. 2015. Emerging resistance against different fungicides in Lasiodiplodia theobromae, the cause of mango dieback in Pakistan. Serbian. Archives Biol. Sci. 67(1):241-249. https://doi.org/10.2298/ ABS140904030R.

Polanco-Florian, L. G.; Alvarado-Gómez, O. G.; Pérez-González, O.; González-Parra, R. y Olivares-Sáenz, E. 2019. Hongos asociados a la muerte regresiva de los cítricos en Nuevo León y Tamaulipas, México. México. Rev. Mex. Cienc. Agríc. 10(4):757-764. https://cienciasagricolas.inifap.gob.mx/index.php/v10n4-01.

Raina, R. 2012. Management of Phytophthora induced foot rot and gummosis of kinnow mandarin in low hills of Himachal Pradesh. Indian. J. Plant Disease Sci. 7(1):9-12. http://www.indianjournals.com/ijor.aspx?target=ijor:jpds\&volume=7\&issue=1\&article $=0$ 02.

Sakalidis, M. L.; Ray, J. D.; Lanoiselet, V.; Hardy, G. E. S. and Burgess, T. I. 2011. Pathogenic Botryosphaeriaceae associated with Mangifera indica in the Kimberley region of Western Australia. Netherlands. Eur. J. Plant Pathol. 130(3):379-391. https://doi.org/10.1007/ s10658-011-9760-z.

Shahbaz, M.; Iqbal, Z.; Saleem, A. and Anjum, M. A. 2009. Association of Lasiodiplodia theobromae with different decline disorders in mango (Mangifera indica L.). Pakistan. Pak. J. Bot. 41(1):359-368. http://www.pakbs.org/pjbot/.

Tovar-Pedraza, J. M.; Mora-Aguilera, J. A.; Nava-Díaz, C.; Teliz-Ortíz, D.; Villegas-Monter, A. y Leyva-Mir, S. G. 2013. Control de Lasiodiplodia theobromae, agente causal de la muerte descendente de injertos de zapote mamey [Pouteria sapota (Jacq.) H. E. Moore y Stearn] en México. México. Rev. Fitotec. Mex. 36(3):233-238. https://www.revistafitotecniamexicana.org/documentos/36-3/6r.pdf.

Valle-Paz, M.; Guillén-Sánchez, D.; Alicia-Tejacal, I.; López-Martínez, V.; Juárez-López, P.; Martínez-Fernández, E.; Hernández-Arenas, M. y Ariza-Flores, R. 2019b. Control in vitro de Lasiodiplodia theobromae (Pat.) Griff. \& Maubl y L. citricola Abdollahz aislados de lima Persa (Citrus latifolia Tanaka) en Morelos, México. México. Acta Agrícola Pecuaria. 5:E0051001. http://riaa.uaem.mx/xmlui/handle/20.500.12055/1159.

Valle-Paz, M.; Guillén-Sánchez, D.; Gijón-Hernández, A. R.; Alía-Tejacal, I.; López-Martínez, V.; Juárez-López, P.; Martínez-Fernández, E.; Hernández-Arenas, M. y Ariza-Flores, R. 2019a. Especies de Lasiodiplodia en lima "Persa" (Citrus latifolia Tanaka) en Morelos, México. México. Revista Bio Ciencias. 6:1-17. https://doi.org/10.15741/revbio.06.e595. 
Wang, H. L.; Chen, P. H.; Ni, H. F. and Chen, R. S. 2007. Physiological characterization and screen of control chemicals for Lasiodiplodia thebromae of papaya. China. Plant Pathology Bulletin. 16(2):71-77. https://www.cabdirect.org/cabdirect/abstract/20073201094.

Yan, J.; Dewdney, M. M.; Roberts, P. D. and Ritenour, M. A. 2016. The effects of postharvest hot water and fungicide treatments on Guignardia citricarpa growth and the development of citrus black spot symptoms on 'Valencia'Orange Fruit. USA. HortScience. 51(12):15551560. https://doi.org/10.21273/HORTSCI11187-16. 